\title{
Lichen planus affecting a 12-year-old girl
}

\author{
Natalie Kelner ${ }^{1}$, Ana Paula Vivas ${ }^{2}$, Rosana Morelatto ${ }^{3}$, Fabio Alves ${ }^{1,2}$ \\ ${ }^{1}$ ACCamargo Hospital, São Paulo, Brazil \\ ${ }^{2}$ University of Sao Paulo, Sao Paulo, Brazil \\ ${ }^{3}$ National University of Córdoba, Córdoba, Brazil \\ Email: nataliekelner@yahoo.com.br
}

Received 21 August 2012; revised 24 September 2012; accepted 9 October 2012

\begin{abstract}
Lichen planus (LP) is a chronic inflammatory mucocutaneous condition which is relatively common in adults but rarely affects children. In addition, few cases with oral lesions affecting children have been reported in the literature. The purpose of this study is to report an unusual case of oral LP involving the tongue and buccal mucosa in a child. A 12-year-old girl complained of painless oral lesions with 18 months of evolution. On clinical evaluation, multiple white lesions in tongue and buccal mucosa were observed with no cutaneous lesions. Diagnosis was made based on clinical examination and histopathological features. No active treatment was necessary. The patient is currently under regular evaluation. Although rarely reported in childhood, lichen planus should be considered in a differential diagnosis of hyperkeratotic and/or reticular lesions of the oral mucosa in children.
\end{abstract}

Keywords: Lichen Planus; Oral; Oral Diagnosis; Child; Diagnosis; Differential

\section{INTRODUCTION}

Lichen planus (LP) is a relatively common chronic inflammatory mucocutaneous condition, which was first described by Eramus Wilson in 1869. The oral lesions are more pleomorphic than those of their cutaneous counterparts and subtypes are categorized as reticular, papular, plaque-like, atrophic, erosive, and bullous. These different clinical presentations represent variations of intensity and duration of the disease. Nowadays, the diagnostic criteria of oral lichen planus (OLP) should include both clinical and histopathological features. Bilateral, somewhat symmetrical lesions with striae are mandatory [1]. The other subtypes, such as erosive, atrophic, bullous and plaque-type, are also found in a reticular pattern. Moreover, the biopsy must present a well-defined bandlike zone of cellular infiltration confined to the superfi- cial part of the connective tissue, signs of liquefaction degeneration of the basal cell layer and absence of epithelial dysplasia [1].

Oral lichen planus in childhood (OLPc) is rare, with few reports available in the literature [2-5]. Predisposing conditions such as graft-versus-host disease, active heaptitis, hepatitis B immunization and autoimmune diseases are frequently mentioned in these reports $[3,4,6,7]$. Furthermore, it is suggested that OLPc is more common in the tropics [8]. In a study of 420 Iranian patients with histopathologic-confirmed OLP, less than $1 \%$ had developed OLP before the age of 13 [9]. This paper reports a rare case of oral lichen planus in a child and discusses the main points on the subject.

\section{CASE REPORT}

A 12-year-old Caucasian girl was referred to the Stomatology Department for evaluation of oral painless lesions with 18 months of evolution. Upon clinical examination, three white distinct lesions were observed. The buccal mucosa and dorsum of the tongue lesions had a typical reticular/striate pattern and a hyperkeratotic pattern was observed in the lateral border of the tongue (Figures 1(a)-(c)).

The patient's oral hygiene was excellent without any dental restorations. However, she was under orthodontic treatment. No other mucosal or skin lesions were present. Our main diagnostic hypotheses were lichen planus and lichenoid lesions. To exclude lichenoid reaction, we investigated her medical status and there was no history of any drug intake. The patient and her parents also denied any habits that may potentially cause oral mucosal reactions. Additionally, we advised to remove the orthodontic brace to exclude lichenoid reactions related to metals. After 40 days, there was little improvement in the clinical inflammatory feature, but the lesions persisted. At this time, an incisional biopsy of the lateral margin of the tongue and of the right buccal mucosa was performed.

Histopathological examination showed hyperparakeratosis of stratified squamous epithelium and basal cell 
degeneration with dense band-linked lymphocytic infiltration at the epithelial-connective tissue interface. There were also some Civatte bodies (Figures 2(a)-(c)). Both clinical and histopathological features were consistent with oral lichen planus. In view of the asymptomatic nature of lichen planus, active treatment was believed to be inappropriate and the patient is being followed periodically (total time of 20 months).

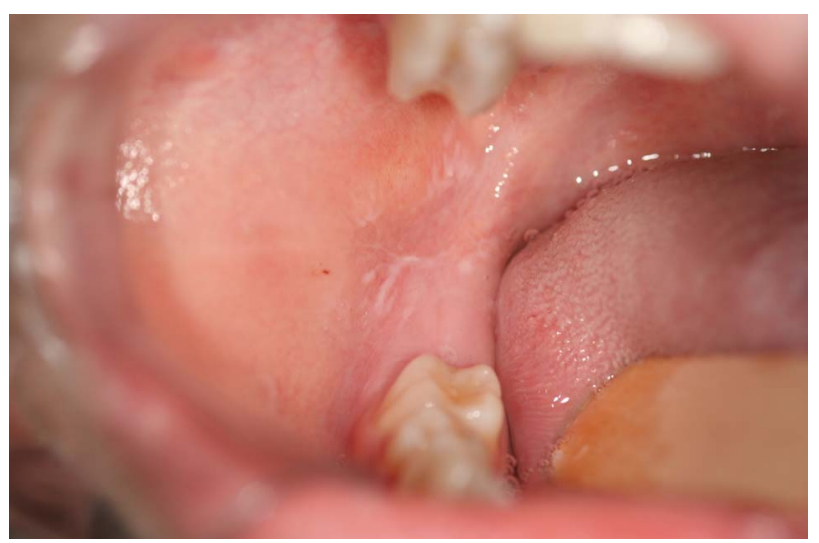

(a)

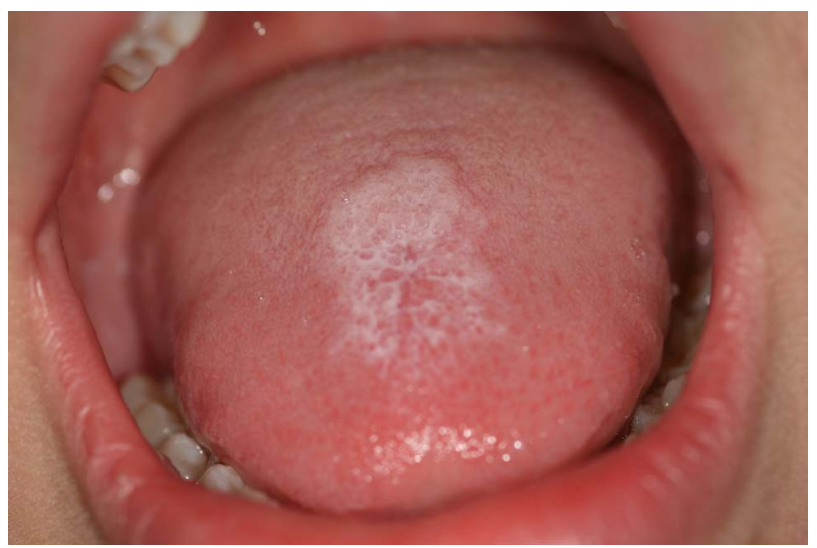

(b)

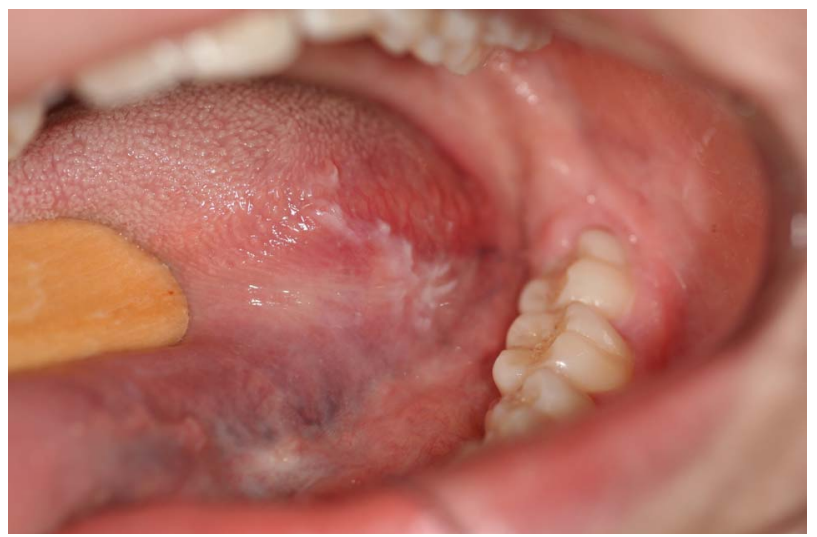

(c)

Figure 1. (a) Reticular pattern in right buccal mucosa; (b) Reticular pattern in dorsum of the tongue; (c) Hyperkeratotic pattern in left border of the tongue.

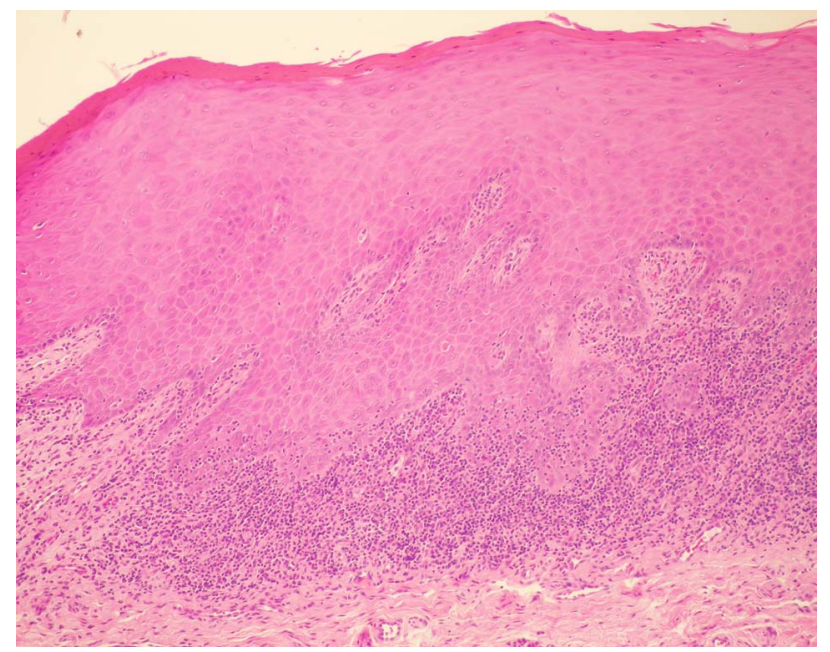

(a)

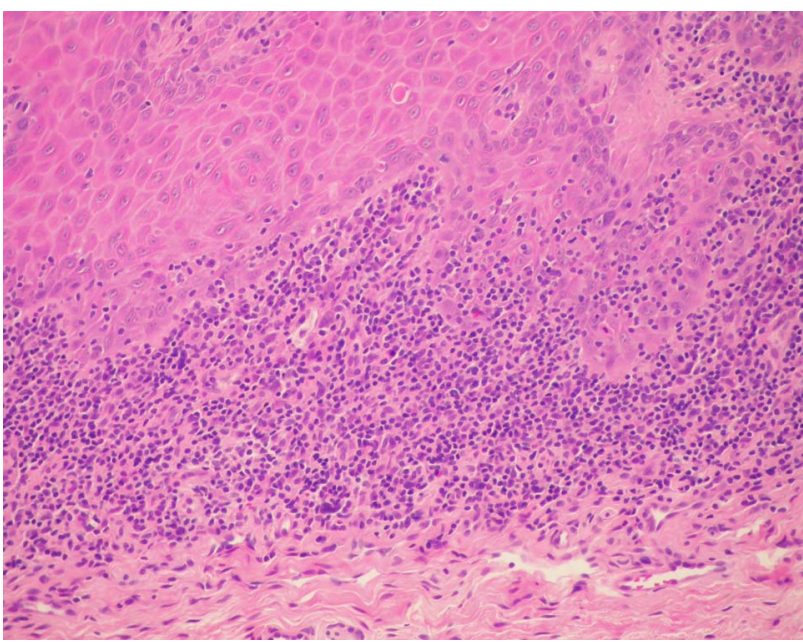

(b)

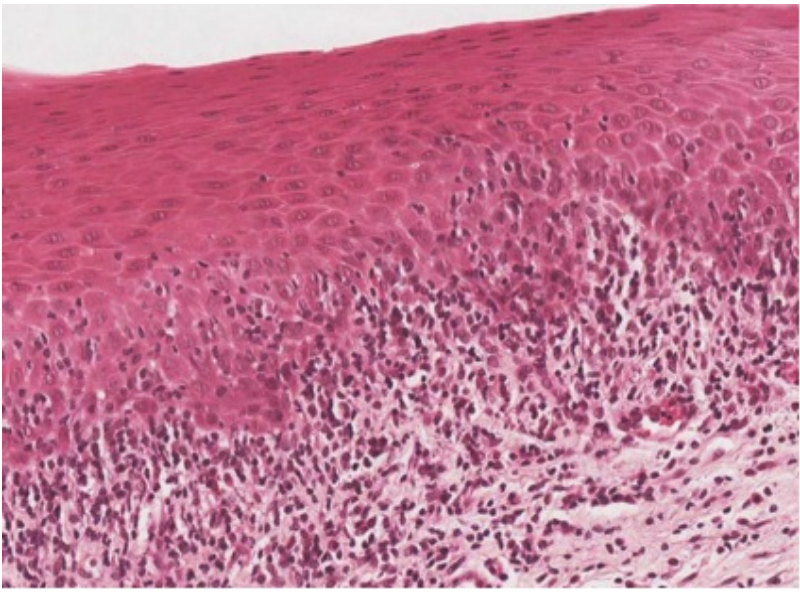

(c)

Figure 2. (a) Histopathological findings. Hyperparakeratosis and dense band-linked inflammatory infiltrate (HE stain 20×); (b) High powered magnification showing an inflammatory infiltrate basically composed by lymphocytes. Some Civatte bodies can also be observed (HE stain $40 \times$ ); (c) High powered magnification showing basal cell layer degeneration (HE stain). 


\section{DISCUSSION}

Lichen planus (LP) is predominately described as a disease of the middle aged or older. Its occurrence in children has been increasingly observed. The global prevalence of LP is lower than $1 \%$ in overall population and the frequency in children can vary between $0.56 \%$ to $13 \%$ of the general lichen planus cases. However, the exact prevalence is still unknown. In an Arabian study, pediatric cases composed 7.5\% of patients with LP [10]. However, it does not appear to be uncommon in Indian/Asian populations. In addition, the largest reported series of lichen planus in childhood (LPc) reported originates from India [11,12]. Even in studies from other countries, some patients were Asian. In a UK study, four out of six OLPc cases reported were from Asian origin [3]. In another UK study, 21 of 26 LPc cases were from the Indian subcontinent [13]. In a study from the Netherlands, two of the three patients with OLPc were from Asian origin, in a region where approximately $1 \%$ of the population is Asian [4]. In another study, Scully et al. (1994) [2] reported three girls with OLPc, one of whom was of Asian origin. Antecedents were investigated in the present case and no Asian relation was found.

Some studies have demonstrated that oral involvement of LP is extremely uncommon in children. Walton et al. (2010) [7] showed that 8 out of 36 children presented oral lesions. Moreover, the largest series of LPc, which evaluated 100 children, 17 of them presented OLP [12]. The clinical features described for OLPc are essentially the same as those in OLP in adults (OLPa), where buccal mucosa, lateral part of the tongue, and the gingival are the main sites affected [2-4]. In adults, LP occurs more commonly in females, but reports of LPc showed discrepancies. Some studies have demonstrated no consistent gender predilection for LPc [14]. In contrast, Walton et al. (2010) [7] showed a female predominance (female to male ratio 2:1) in 36 patients. However, in large series [11-13], boys have been shown to outnumber girls. In addition, all 6 cases reported by Alam and Hamburger (2001) [3], were boys. The case presented here, a 12year-old girl, had lesions affecting the dorsum and lateral border of the tongue and buccal mucosa.

There is a spectrum of oral lichenoid lesions (OLL) that can determine a differential diagnosis of OLP. OLL encompass several clinical settings: 1) Oral lichenoid contact lesions as a result of allergic contact stomatitis. The lesions are seen in close contact to dental restorative materials, most commonly amalgam, or other contacted agents; 2) Oral lichenoid drug reaction, wherein oral and/or skin lesions arise in temporal association with taking of certain medications, such as oral hypoglycemic agents, angiotensin-converting enzyme inhibitors, and nonsteroidal anti-inflammatory agents; 3) Oral liche- noid lesions of graft-versus-host disease, a common complication of allogeneic hematopoietic stem-cell or bone marrow transplantation [15].

The elimination of factors that may precipitate or provoke oral lesions is an important initial step in diagnosis of oral lichen planus. Our patient had no relevant medical or family history, nor presented any deleterious oral habits and her oral hygiene was excellent, without any dental restorations. She was under orthodontic treatment, and consequently our first differential diagnosis was of lichenoid reaction, which was later ruled out after the orthodontic brace had been removed. The differential diagnosis of OLPc may be quite extensive and, of course, depends on the age of the patient, the clinical variant, and the severity and the persistence of the lesions and includes candidosis, morsicatio buccarum, leucoplakia, stomatitis migrans, auto-immune bullous diseases, lupus erythematosus, several viral infections (herpes simplex, Epstein-Barr, Coxsackie, HIV), recurrent aphthous stomatitis (caustic) traumata, erythema multiforme (major), allergic gingivostomatitis, gluten sensitivity enteropathy, and less commonly Crohn and Behçet diseases, oral lesions in immunodeficiencies, dyskeratosis follicularis, pachyonychia congenita, dyskeratosis congenita, white sponge nevus; allergy to flavorings may be relevant instead in the diagnosis of allergic contact stomatitis [16].

Clinically and histopathologically, OLL may be indistinguishable from OLP. However, the distinguishing features of oral lichenoid contact lesions is the lesion's direct topographic relationship to the suspected causative agent, sites that have a direct contact with filling/dental restorations or another offending contact agent (e.g. cinnamon). Removal of the suspected causative factors should result in resolution of the lesion, thereby establishing the diagnosis [15].

The treatment of LPc consists of topical corticosteroids and oral histamines [11]. For oral lichen planus, topical treatment options including corticosteroids in orabase, topical tretinoin, isotretinoin gel and tacrolimus or pimecrolimus have been prescribed [4,11-12,15]. Oral agents such as systemic glucocorticoids, griseofulvin, hydroxychloroquine, azathioprine, mycophenolate mofetil and triamcinolone have also been described in the literature $[4,12,15]$. The majority of children affected with oral lichen planus have been asymptomatic. Our case was asymptomatic, therefore, no active treatment was provided.

The prognosis and the effect of treatment in OLPc seems to be more favorable than in OLPa, which usually persists for many years in spite of intensive treatment and thorough investigation of associated factors [3,5]. Malignant transformation of OLPa, especially the erosive variety, has been documented, with about $0.07 \%$ to $5 \%$ $[5,9]$. However, to our knowledge, no malignant trans- 
formation of OLPc has yet to be reported.

It is not possible to draw firm conclusions about many of the aspects of OLPc especially associated factors, treatment, prognosis, age of preference, and the boy-togirl ratio, because of the low numbers of children affected with OLP. Nevertheless, the schedule of follow-up of OLPC and also the removal of local irritant factors should number at least one or two visits per year, as long as OLPc persists and even more frequently in symptommatic ones, in line with OLPa. Although oral lichen planus in childhood is rare, this diagnosis should be considered in children presenting with oral white lesion.

\section{REFERENCES}

[1] Van der Meij, E.H. and Van der Waal, I. (2003) Lack of clinicopathologic correlation in the diagnosis of oral lichen planus based on the presently available diagnostic criteria and suggestions for modifications. Journal of Oral Pathology and Medicine, 32, 507-512. doi:10.1034/j.1600-0714.2003.00125.x

[2] Scully, C., de Almeida, O.P. and Wellbury, R. (1994) Oral lichen planus in childhood. British Journal of Dermatology, 134, 131-133. doi:10.1111/j.1365-2133.1994.tb06905.x

[3] Alam, F. and Hamburger, J. (2001) Oral mucosal lichen planus in children. International Journal of Paediatric Dentistry, 11, 209-214. doi:10.1046/j.1365-263X.2001.00266.X

[4] Laeijendecker, R., Joost, T.V., Tank, B., et al. (2005) Oral lichen planus in childhood. Pediatric Dermatology, 22, 299-304. doi:10.1111/j.1525-1470.2005.22403.x

[5] Eisen, D. (2002) The clinical features, malignant potential, and systemic associations of oral lichen planus: A study of 723 patients. Journal of the American Academy of Dermatology, 46, 207-214. doi:10.1067/mjd.2002.120452

[6] Cottoni, F., Ena, P., Tedde, G., et al. (1993) Lichen planus in children: A case report. Pediatric Dermatology,
10, 132-135. doi:10.1111/j.1525-1470.1993.tb00038.x

[7] Walton, K.E., Bowers, E.V., Drolet, B.A., et al. (2010) Childhood lichen planus: Demographics of US population. Pediatric Dermatology, 27, 34-38. doi:10.1111/j.1525-1470.2009.01072.x

[8] Ramsay, D.L. and Hurley, H.J. (1985) Papulosquamous eruptions and exfoliative dermatitis. In: Moschella, S.L. and Hurley, H.J., Eds., Dermatology, Vol. 1, 2nd Edition, W.B. Saunders, Philadelphia, 529-535.

[9] Pakfetrat, A., Javadzadeh-Bolouri, A., Basir-Shabestari, S., et al. (2009) Oral lichen planus: A retrospective study of 420 Iranian patients. Medicina Oral Patologia Oral y Cirurgia Bucal, 14, E315-E318.

[10] Kanwar, A.J. and Belhaj, M.S. (1984) Lichen planus among Arabs-A study from Libya. The Journal of Dermatology, 11, 93-96.

[11] Sharma, R. and Maheshwari, V. (1999) Childhood lichen planus: A report of fifty cases. Pediatric Dermatology, 16, 345-348. doi:10.1046/j.1525-1470.1999.00074.x

[12] Kanwar, A.J. and D. De (2010) Lichen planus in childhood: Report of 100 cases. Clinical and Experimental Dermatology, 35, 257-262. doi:10.1111/j.1365-2230.2009.03613.x

[13] Balamasubramanian, P., Ogboli, M. and Moss, C. (2008) Lichen planus in children: Review of 126 cases. Clinical and Experimental Dematology, 33, 457-459. doi:10.1111/j.1365-2230.2008.02694.x

[14] Handa, S. and Sahho, B. (2002) Childhood lichen planus: A study of 87 cases. International Journal of Dermatology, 41, 423-427. doi:10.1046/j.1365-4362.2002.01522.x

[15] Al-Hashimi, I., Schifter, M., Lockhart, P.B., et al. (2007) Oral lichen planus and oral lichenoid lesions: Diagnostic and therapeutic considerations. Oral Surgery, Oral Medicine, Oral Pathology, Oral Radiology, and Endodontology, 103, e1-e12.

[16] Yiannias, J.A., El-Azhary, R.A., Hand, J.H., et al. (2000) Relevant contact sensitivities in patients with the diagnosis of oral lichen planus. Journal of the American Academy of Dermatology, 42, 177-182. doi:10.1016/S0190-9622(00)90123-3 